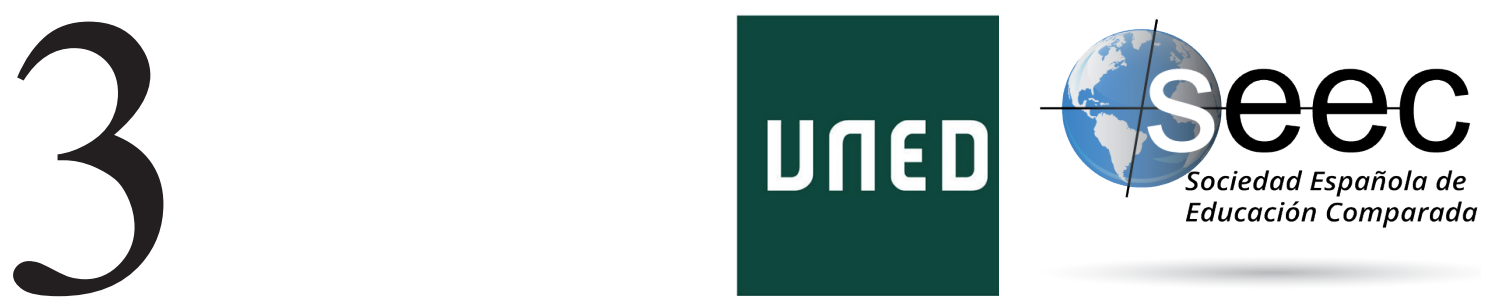

\title{
El máster universitario en España y las opciones de los estudiantes chinos
}

\author{
The master 's degree in Spain and \\ the Chinese students' choice
}

\section{Zhang Menglu*}

DOI: $10.5944 /$ reec.31.2018.20171

Recibido: 25 de octubre de 2017 Aceptado: 4 de abril de 2018

**Zhang Menglu: Doctora. Profesora de la Facultad de Español del Departamento de Lenguas y Culturas Occidentales de la Universidad de Estudios Extranjeros de Guangdong, Guangzhou, República Popular China. Está especializada en teoría y práctica de interpretación chino-español y pedagogía. Datos de contacto: E-mail: menglu711@sina.com 


\title{
Resumen
}

La educación de máster universitario en España es bien conocida por su larga historia, amplia gama de especialidades, asignaturas diferenciadas y métodos de enseñanza flexibles. Partiendo del análisis del corpus de los certificados de máster universitario obtenidos por estudiantes chinos, el artículo presenta objetivamente la distribución universitaria, el diseño de especialidades y el programa de máster de las universidades españolas que tienen alumnos chinos. Al mismo tiempo, hace una comparación con la educación de máster en China. El objetivo de nuestra investigación es dar a conocer los motivos por los cuales los postgrados de España atraen cada vez más a los estudiantes chinos y mostrar el desarrollo de los programas de máster en China.

Palabras clave: universidades españolas; estudiantes chinos; máster universitario; especialidad; asignatura

\begin{abstract}
The master's education in Spain is well known for its long history, wide range of specialties, differentiated subjects and flexible teaching methods. Based on the analysis of the corpus of university master's certificates obtained by Chinese students, the article presents objectively the university distribution, the design of specialties and the curricular of the máster of the Spanish universities that have Chinese students. At the same time, it makes a comparison with the máster's education in China. The intention of our research is to publicize the reasons why the postgraduate courses in Spain attract more and more Chinese students and show the development of master's programs in China.
\end{abstract}

Key Words: Spanish universities; Chinese students; máster's degree; specialty; subjects 


\section{Introducción}

La educación superior española tiene una larga historia y un sistema integral. Según el Ministerio de Educación de España, los estudios universitarios en el Estado español se distribuyen en 3 ciclos: el primer ciclo es el grado (la antigua licenciatura); el segundo ciclo es el máster; el tercer ciclo es el doctorado ${ }^{1}$ :

Los estudios de grado constan de un total de 240 créditos europeos, normalmente distribuidos en cuatro años. Solo para ejercer determinadas profesiones que presentan características particulares, como abogado, profesor de educación secundaria o ingeniero, los graduados tendrán que estudiar un máster.

Los estudios de máster comprenden entre 60 y 120 créditos europeos (entre 1 y 2 años). Existen 3 tipos de máster: máster orientado a la especialización; máster necesario para ejercer una profesión y máster orientado a la investigación.

El doctorado es el tercer ciclo de las enseñanzas universitarias y se compone de un período de formación y un período de investigación. Esta última etapa finaliza con la elaboración de una tesis doctoral.

Aunque la escala de matrícula universitaria no es tan grande como en China, las universidades españolas tienen un alto nivel de internacionalización, especialmente en la etapa de formación de máster/postgrado. Algunas disciplinas y especialidades de universidades españolas están atrayendo cada día a más estudiantes extranjeros. Los datos e informaciones del Centro de Servicio para Intercambio Académico de China ${ }^{2}$ muestran que, solo en 2016, más de 200 estudiantes chinos obtuvieron títulos oficiales de máster universitario con una cobertura de más de 120 especialidades. La fecha de graduación oscila entre junio de 2011 y marzo de 2017. Este artículo analiza la distribución universitaria, especialidades y asignaturas para conocer el plan de estudios, sistema de materias y necesidades de los estudiantes basándose en el corpus de títulos y certificados de máster universitario obtenidos por estudiantes chinos. Con esta investigación empírica, pretendemos proporcionar una referencia para mejorar aún más tanto la educación superior como los cursos de máster de China.

\section{Universidades españolas elegidas por estudiantes chinos}

De acuerdo con las últimas estadísticas, existen 82 universidades en España, entre ellas, 50 públicas y 32 privadas ${ }^{3}$. En 2012, España, junto con los Estados Unidos, Gran Bretaña, Francia y China, es conocida como uno de los diez países mundiales de destino de estudio según la Organización Mundial de Cooperación y Desarrollo Económicos (OCDE). De los 217 estudiantes chinos encuestados que han obtenido títulos oficiales de máster universitario en España, 24 han elegido universidades públicas, dos de ellos universidades privadas, y otros dos, institutos de investigación en España. Véase la siguiente tabla y figura:

1 http://www.educacion.gob.es/boloniaensecundaria/o9-damos-respuesta1.htm\#pregunta2

2 Desde enero de 2016, la autora empezó a trabajar como traductora de títulos y certificados de máster universitario de España logrados por estudiantes chinos en el Centro de Servicio para el Intercambio Académico de China. Este trabajo es una investigación empírica basada en el corpus de los títulos y certificados.

3 http://www.universia.es/universidades

4 http://bg.yjbys.com/diaochabaogao/22303.html 


\section{Tabla 1}

Universidades españolas elegidas por estudiantes chinos

\begin{tabular}{|c|c|c|c|c|}
\hline NO. & Universidad & $\begin{array}{c}\text { Ubicación } \\
\text { (Comunidad } \\
\text { Autónoma) } \\
\end{array}$ & & $\begin{array}{l}\text { Número de } \\
\text { estudiantes } \\
\text { chinos }\end{array}$ \\
\hline 1 & Universidad Rey Juan Carlos & Comunidad de Madrid & Público & 30 \\
\hline 2 & $\begin{array}{l}\text { Universitat Autònoma } \\
\text { de Barcelona }\end{array}$ & Cataluña & Público & 27 \\
\hline 3 & $\begin{array}{l}\text { Universidad Complutense } \\
\text { de Madrid }\end{array}$ & Comunidad de Madrid & Público & 26 \\
\hline 4 & Universidad Carlos III de Madrid & Comunidad de Madrid & Público & 20 \\
\hline 5 & $\begin{array}{l}\text { Universidad de Alcalá } \\
\text { de Henares }\end{array}$ & Comunidad de Madrid & Público & 18 \\
\hline 6 & Universidad de Sevilla & Andalucía & Público & 10 \\
\hline 7 & Universidad de Salamanca & Castilla y León & Público & 10 \\
\hline 8 & $\begin{array}{l}\text { Universidad Autónoma } \\
\text { de Madrid }\end{array}$ & Comunidad de Madrid & Público & 9 \\
\hline 9 & Universidad de Granada & Andalucía & Público & 8 \\
\hline 10 & Universidad de Barcelona & Cataluña & Público & 6 \\
\hline 11 & Universidad de Valencia & Comunidad Valenciana & Público & 6 \\
\hline 12 & $\begin{array}{l}\text { Universidad Politécnica } \\
\text { de Madrid }\end{array}$ & Comunidad de Madrid & Público & 5 \\
\hline 13 & Universidad Ramón Llull & Cataluña & Privado & 5 \\
\hline 14 & Universidad Rovira i Virgiri & Cataluña & Público & 5 \\
\hline 15 & Universidad de Málaga & Andalucía & Público & 4 \\
\hline 16 & $\begin{array}{l}\text { Universidad Politécnica } \\
\text { de Valencia }\end{array}$ & Comunidad Valenciana & Público & 4 \\
\hline 17 & Universidad Pompeu Fabra & Cataluña & Público & 4 \\
\hline 18 & Universidad de Alicante & Comunidad Valenciana & Público & 4 \\
\hline 19 & $\begin{array}{l}\text { Universidad Politécnica } \\
\text { de Cataluña }\end{array}$ & Cataluña & Público & 3 \\
\hline 20 & Universidad de Burgos & Castilla y León & Público & 2 \\
\hline 21 & Universidad de Valladolid & Castilla y León & Público & 2 \\
\hline 22 & Universidad de Oviedo & Asturias & Público & 2 \\
\hline 23 & Universidad de Cádiz & Andalucía & Público & 2 \\
\hline 24 & Universidad de Jaén & Andalucía & Público & 1 \\
\hline 25 & Universidad de las Illes Balears & Islas Baleares & Público & 1 \\
\hline 26 & Universidad Pontificia Comillas & Comunidad de Madrid & Privado & 1 \\
\hline 27 & $\begin{array}{l}\text { IED Madrid Centro Superior } \\
\text { de Diseño }\end{array}$ & Comunidad de Madrid & Privado & 1 \\
\hline 28 & Instituto de Empresa & Comunidad de Madrid & Privado & 1 \\
\hline Total & & & & 217 \\
\hline
\end{tabular}




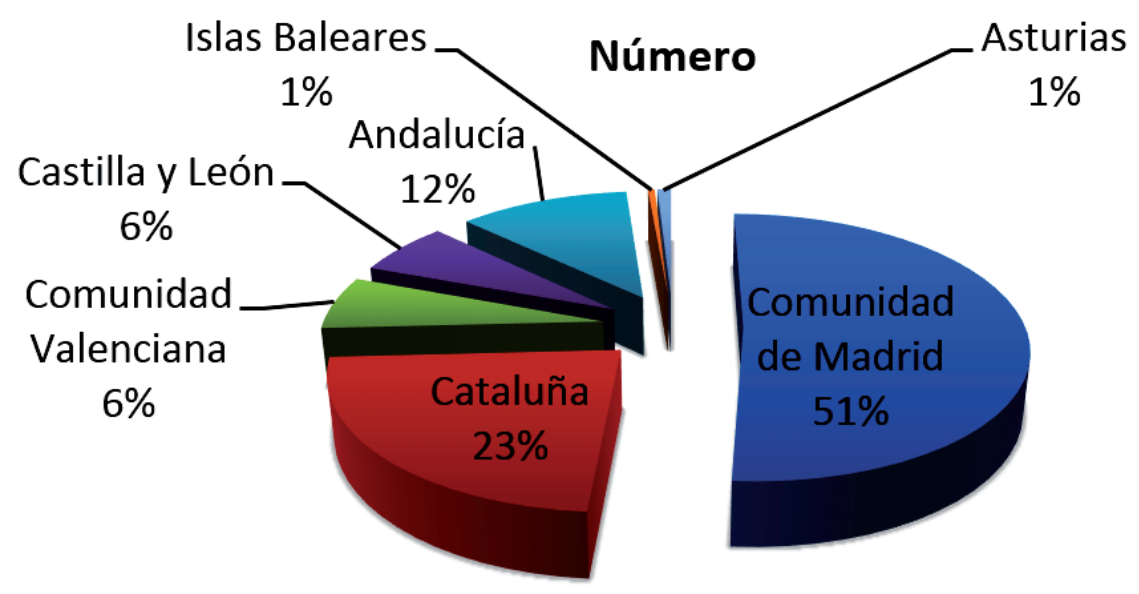

Figura 1. Distribución regional universitaria.

En la figura 1 podemos ver que la mayoría de los estudiantes chinos logran títulos de máster universitario en las grandes ciudades de la Comunidad de Madrid (51\%) y Cataluña (23 \%), seguidas por las ciudades de Andalucía (12 \%), Castilla y León (6 \%) y la Comunidad Valenciana (6 \%). Pocos estudiantes eligen universidades en el norte, por ejemplo: Asturias (1\%) y en zonas insulares como Islas Baleares (1\%). Se nota un desequilibrio de distribución de estudiantes chinos. Para resolver este problema, Quiroga (2017:476) plantea que hace falta una mayor expansión del intercambio entre universidades españolas y chinas, para que más estudiantes chinos estudien en diferentes zonas españolas. Por otra parte, el 96,3 \% opta por máster de universidades públicas, y solo 8 personas ( $3 \%$ ) seleccionan universidades e instituciones privadas.

Partiendo de los datos anteriores, podemos conocer que los alumnos chinos estudian másteres tanto en universidades con larga historia como en universidades relativamente jóvenes. Por ejemplo, la Universidad Complutense de Madrid (1293) y la Universidad de Alcalá (1499); la Universidad Autónoma de Madrid (1968), y la Universidad de Carlos III de Madrid (1989), etc. Cada una de estas universidades tiene sus propias características en asignaturas, recursos docentes e intercambios internacionales. Por ejemplo, la Universidad Complutense de Madrid es una de las más prestigiosas tanto en el país como en el mundo. Durante muchos años, ocupa el primer lugar del ranking de universidades españolas ${ }^{5}$. Hoy en día, es la universidad más grande y más completa de España, que abarca principalmente cinco disciplinas: humanidades, ciencias, ciencias de la salud, ciencias sociales y jurídicas, ingenierías y arquitectura; así como 175 especialidades de másteres para el curso 2017-186. La Universidad Carlos III de Madrid se fundó en 1989 y fue la primera universidad de España en incorporar todos los programas de grado, máster y doctorado en el programa Erasmus. ${ }^{7}$ Debido a eso, ha registrado un avance

5 http://www.elmundo.es/ranking-universidades.html

6 http://www.ucm.es/estudios/máster

7 El programa Erasmus, acrónimo del nombre oficial en idioma inglés European Region Action Schemeforthe Mobility of University Students (Plan de Acción de la Comunidad Europea para la Movilidad de Estudiantes Universitarios), es un plan de gestión de diversas administraciones públicas por el que se apoya y facilita la movilidad académica de los estudiantes y profesores universitarios dentro de los Estados miembros del Espacio Económico Europeo, Suiza y Turquía. Disponible en https://es.wikipedia.org/wiki/Programa_Erasmus 
muy notable y se sitúa en el octavo lugar en el ranking de universidades españolas ${ }^{8}$. En la actualidad, muchos estudiantes chinos prefieren ir allí para estudiar. En lo que va el año, una veintena de alumnos han logrado títulos oficiales de máster universitario de esta Universidad. Para el año 2018, según el ranking mundial QS9 , la Universidad Complutense ocupa el $4^{\circ}$ lugar de España y la Universidad Carlos III el $7^{\circ}$.

\section{Disciplinas y especialidades preferidas por los estudiantes chinos}

Los estudiantes chinos prefieren los estudios de máster en España por los programas científicos, las asignaturas relacionadas con la cultura española, las especialidades adaptadas a las necesidades sociales y al desarrollo del momento.

Desde la década de 1990, la proporción del máster de humanidades, ciencias sociales y jurídicas en el mundo (región) ha mostrado una tendencia creciente en comparación con el máster de ciencias (Jianlin, 2009:66). A través de la tabla 2, descubrimos que solo 27 estudiantes chinos eligen el máster de ciencias, ciencias de la salud, ingenierías y arquitectura, representando el $13 \%$ de la totalidad.

\section{Tabla 2}

Másteres de ciencias, ciencias de la salud, ingenierías y arquitectura elegidos por los estudiantes chinos

\begin{tabular}{|c|c|c|c|}
\hline Universidad & Especialidad & Disciplina & $\begin{array}{l}\text { Número } \\
\text { de estudiantes } \\
\text { chinos }\end{array}$ \\
\hline \multirow{2}{*}{$\begin{array}{l}\text { Universidad } \\
\text { Complutense } \\
\text { de Madrid } \\
\end{array}$} & Geología Ambiental y Recursos Geológicos & \multirow{10}{*}{$\begin{array}{l}\text { Ingenierías y } \\
\text { arquitectura }\end{array}$} & 1 \\
\hline & Energía & & 1 \\
\hline $\begin{array}{l}\text { Universidad } \\
\text { Carlos III } \\
\text { de Madrid } \\
\end{array}$ & Ingeniería Matemática & & 1 \\
\hline \multirow{4}{*}{$\begin{array}{l}\text { Universidad } \\
\text { Politécnica } \\
\text { de Madrid }\end{array}$} & Planificación Urbana y Regional & & 1 \\
\hline & Proyectos Arquitectónicos Avanzados & & 1 \\
\hline & Estructuras de la Edificación & & 1 \\
\hline & Ingeniería Mecánica & & 1 \\
\hline \multirow{3}{*}{$\begin{array}{l}\text { Universidad } \\
\text { Politécnica } \\
\text { de Cataluña }\end{array}$} & Ingeniería Civil & & 1 \\
\hline & $\begin{array}{l}\text { Teoría y Práctica del Proyecto } \\
\text { de Arquitectura }\end{array}$ & & 1 \\
\hline & Ciencia y Tecnología Aeroespacial & & 1 \\
\hline
\end{tabular}

(Continua en la siguiente página)

\footnotetext{
8 http://www.elmundo.es/ranking-universidades.html

9 http://www.lavanguardia.com/vida/20170607/423254024982/comunicado-qs-world-universityrankings-2018.html
} 


\begin{tabular}{|c|c|c|c|}
\hline Universidad & Especialidad & Disciplina & $\begin{array}{l}\text { Número } \\
\text { de estudiantes } \\
\text { chinos }\end{array}$ \\
\hline $\begin{array}{l}\text { Universidad } \\
\text { de Burgos }\end{array}$ & $\begin{array}{l}\text { Cultura del Vino: Enoturismo en la Cuenca } \\
\text { del Duero }\end{array}$ & \multirow{4}{*}{ Ciencias } & 2 \\
\hline $\begin{array}{l}\text { Universidad } \\
\text { de Salamanca }\end{array}$ & $\begin{array}{l}\text { TIC en Educación: Análisis y Diseño de } \\
\text { Procesos, Recursos y Prácticas Formativas }\end{array}$ & & 2 \\
\hline $\begin{array}{l}\text { Universidad } \\
\text { Politécnica de } \\
\text { Valencia }\end{array}$ & Automática e Informática Industrial & & 1 \\
\hline $\begin{array}{l}\text { Universidad } \\
\text { de Oviedo }\end{array}$ & Biotecnología Alimentaria & & 1 \\
\hline \multirow{2}{*}{$\begin{array}{l}\text { Universidad } \\
\text { de Granada }\end{array}$} & Óptica y Optometría Avanzada & \multirow{4}{*}{$\begin{array}{l}\text { Ciencias de } \\
\text { la salud }\end{array}$} & 2 \\
\hline & $\begin{array}{l}\text { Avances en Radiología Diagnóstica } \\
\text { y Terapéutica y Medicina Física }\end{array}$ & & 5 \\
\hline $\begin{array}{l}\text { Universidad } \\
\text { de Sevilla }\end{array}$ & $\begin{array}{l}\text { Investigación Médica: Clínica } \\
\text { y Experimental }\end{array}$ & & 5 \\
\hline $\begin{array}{l}\text { Universidad } \\
\text { de Valencia }\end{array}$ & Ciencias Odontológicas & & 1 \\
\hline \multicolumn{3}{|l|}{ Total } & 29 \\
\hline
\end{tabular}

Analizando los datos de estos estudiantes podemos descubrir que las especialidades de ciencias incluyen disciplinas o materias relacionadas con la historia y cultura de España como «Cultura del Vino: Enoturismo en la Cuenca del Duero» y otras de las áreas emergentes de la ciencia y la tecnología, como «Ciencia y Tecnología Aeroespacial», proporcionando un amplio espacio para que los estudiantes chinos puedan seleccionar.

\subsection{Diversidad de especialidades de másteres de humanidades, ciencias sociales y jurídicas}

Entre los estudiantes chinos encuestados, el $23 \%$ elige la especialidad relacionada con la filología hispánica. Sobre este ámbito, algunas universidades disponen de más de una especialidad. Véase la siguiente tabla:

\section{Tabla 3}

Especialidad de la lengua española y opción de los estudiantes chinos

\begin{tabular}{|c|c|c|c|}
\hline & Especialidad & Universidad & $\begin{array}{c}\text { Número } \\
\text { de estudiantes } \\
\text { chinos }\end{array}$ \\
\hline 1 & $\begin{array}{l}\text { Lengua Española, Literatura Hispánica } \\
\text { y Español como Lengua Extranjera }\end{array}$ & \multirow{4}{*}{$\begin{array}{l}\text { Universidad Autónoma } \\
\text { de Barcelona }\end{array}$} & 2 \\
\hline 2 & $\begin{array}{l}\text { Traducción, Interpretación } \\
\text { y Estudios Interculturales }\end{array}$ & & 8 \\
\hline 3 & $\begin{array}{l}\text { Tratamiento de la Información } \\
\text { y Comunicación Multilingüe }\end{array}$ & & 2 \\
\hline 4 & $\begin{array}{l}\text { Lengua Española, Literatura Hispánica } \\
\text { Español como Lengua Extranjera }\end{array}$ & & 1 \\
\hline
\end{tabular}




\begin{tabular}{|c|c|c|c|}
\hline & Especialidad & Universidad & $\begin{array}{c}\text { Número } \\
\text { de estudiantes } \\
\text { chinos }\end{array}$ \\
\hline 5 & $\begin{array}{l}\text { Enseñanza del Español } \\
\text { como Lengua Extranjera }\end{array}$ & \multirow{2}{*}{$\begin{array}{l}\text { Universidad } \\
\text { Complutense de Madrid }\end{array}$} & 3 \\
\hline 6 & $\begin{array}{l}\text { Pensamiento Español } \\
\text { e Iberoamericano }\end{array}$ & & 3 \\
\hline 7 & $\begin{array}{l}\text { Español y Literatura } \\
\text { Hispánica Contemporánea }\end{array}$ & \multirow{2}{*}{$\begin{array}{l}\text { Universidad } \\
\text { Carlos III de Madrid }\end{array}$} & 4 \\
\hline 8 & Literatura Hispánica Contemporánea & & 5 \\
\hline 9 & $\begin{array}{l}\text { Comunicación Intercultural, Inter- } \\
\text { pretación y Traducción } \\
\text { en los Servicios Públicos } \\
\text { Especialidad en Chino-Español }\end{array}$ & Universidad de Alcalá & 8 \\
\hline 10 & $\begin{array}{l}\text { Traducción y Comunicación Inter- } \\
\text { cultural }\end{array}$ & Universidad de Sevilla & 2 \\
\hline 11 & $\begin{array}{l}\text { Lengua Española: Investigación } \\
\text { y Prácticas Profesionales }\end{array}$ & \multirow{3}{*}{$\begin{array}{l}\text { Universidad Autónoma } \\
\text { de Madrid }\end{array}$} & 1 \\
\hline 12 & $\begin{array}{l}\text { Pensamiento Español } \\
\text { e Iberoamericano }\end{array}$ & & 2 \\
\hline 13 & $\begin{array}{l}\text { Literatura Hispánica: Arte, Historia } \\
\text { y Sociedad }\end{array}$ & & 2 \\
\hline 14 & $\begin{array}{l}\text { Experto en Español como Lengua } \\
\text { Extranjera en Ámbitos profesionales }\end{array}$ & Universidad de Barcelona & 1 \\
\hline 15 & $\begin{array}{l}\text { Estudios Hispánicos: } \\
\text { Aplicaciones e Investigación }\end{array}$ & Universidad de Valencia & 1 \\
\hline 16 & $\begin{array}{l}\text { Enseñanza de Lenguas Extranjeras } \\
\text { (Español Lengua Extranjera/Inglés } \\
\text { Lengua Extranjera) }\end{array}$ & $\begin{array}{l}\text { Universidad Rovira I } \\
\text { Virgili }\end{array}$ & 2 \\
\hline 17 & Estudios de Traducción & \begin{tabular}{|l|} 
Universidad \\
Pompeu Fabra \\
\end{tabular} & 1 \\
\hline 18 & $\begin{array}{l}\text { Enseñanza del Español como Lengua } \\
\text { Extranjera }\end{array}$ & \begin{tabular}{|l|} 
Universidad \\
de Valladolid
\end{tabular} & 1 \\
\hline Total & & & 49 \\
\hline
\end{tabular}

Aparte de la filología hispánica, el máster universitario en administración, liderazgo y gestión también incluye varias especialidades. 50 estudiantes chinos han conseguido títulos de este ámbito. En sus títulos aparecen 16 denominaciones diferentes. Por ejemplo: Gestión de Personal, Dirección de Recursos Humanos en Organizaciones, Gestión Empresarial, Técnicas Cuantitativas en Gestión Empresarial, Dirección de Comunicación Empresarial e Institucional, Gestión, Organización y Economía de la Empresa, Gestión Administrativa del Comercio Internacional, Administración de Empresas Internacionales, Dirección y Administración de Empresas, Administración de Empresas Turísticas, Gestión de Liderazgo, Gestión de Cultura, Gestión de Proyectos Culturales, Gestión de la Documentación, Bibliotecas y Archivos, etc. 


\subsection{Surgimiento de especialidades de las ciencias e interdisciplinarias}

En el marco de las ciencias, también aparece una serie de carreras emergentes y de alto nivel tecnológico. Por ejemplo, Energía Solar Fotovoltaica de la Universidad Politécnica de Madrid, Biotecnología Alimentaria de la Universidad de Oviedo, Ciencia y Tecnología Aeroespacial de la Universidad Politécnica de Cataluña, así como otras carreras relacionadas con el ámbito más reciente de la ciencia y la tecnología. Por otro lado, se han iniciado nuevas especialidades interdisciplinarias, tales como: Cine, Televisión y Medios Interactivos de la Universidad Rey Juan Carlos, Iniciativa Emprendedora y Creación de Empresas de la Universidad Carlos III, Turismo Electrónico: Tecnologías Aplicadas a la Gestión y Comercialización del Turismo de la Universidad de Málaga, Técnicas de Análisis e Innovación Turística de la Universidad Rovira i Virgili, Economía del Turismo y Medio Ambiente de la Universidad de Les Illes Balears, etc. Cada día hay más estudiantes chinos que optan por estas carreras y han logrado títulos oficiales de máster universitario.

\subsection{Especialidades exclusivas en España}

Algunos estudiantes chinos eligen especialidades que solo existen en máster en España. Por ejemplo: Cultura del Vino, Patrimonio Cultural: Identificación, Análisis y Gestión; Dirección y Gestión de Producto de Moda; Didácticas Específicas en el Aula, Museos y Espacios Naturales, etc. Estas especialidades cuentan con peculiaridades culturales y históricas españolas.

\subsection{Títulos conjuntos nacionales e internacionales de máster universitario}

Las universidades pueden, mediante convenio con otras universidades nacionales o extranjeras, organizar enseñanzas conjuntas conducentes a la obtención de un único título oficial de máster universitario ${ }^{10}$. Por otra parte, en el proceso de la integración de la educación superior de la UE, España responde positivamente a la iniciativa del «Programa Erasmus» ${ }^{11}$ y fomenta el intercambio nacional e internacional de la educación superior. Esta tendencia también se refleja en el diseño de especialidades de máster universitario para estudiantes extranjeros, cuyos modelos se dividen en 3 tipos:

Primero, «títulos conjuntos universitarios». Según el corpus, un estudiante chino eligió el título de Máster en Gestión de Economía e Innovación establecido por la Universidad Complutense de Madrid, la Universidad Autónoma de Madrid y la Universidad Politécnica de Madrid. Se trata de un título conjunto de máster otorgado por 3 universidades de la misma comunidad autónoma.

Segundo, «títulos conjuntos nacionales». Un estudiante chino optó por estudiar el Máster Universitario en Condiciones Genéticos, Nutricionales y Ambientales del Crecimiento y Desarrollo establecido por la Universidad de Granada, la Universidad de Santiago de Compostela, la Universidad de Zaragoza, la Universidad de Rovira i Virgili y la Universidad de Cantabria. Se trata de un título conjunto de máster otorgado por 5 universidades de España.

Tercero, «títulos conjuntos internacionales». En cuanto a la cooperación internacional, España ha establecido el «máster Europeo», por lo que se convierte en el destino más popular entre los estudiantes europeos. Por ejemplo, entre los 58 títulos de máster universitario de la Universidad de Cataluña, 10 pertenecen a «máster Europeo» (Xiuyan,

\footnotetext{
10 http://servicio.us.es/academica/titulos-conjuntos-de-grado-y-máster

11 El Programa Erasmus se estableció en 1987 por la Comunidad Europea para promover el intercambio de estudiantes dentro de la Comunidad Europea.
} 
2013:79). Sin embargo, esta cooperación no se limita a universidades europeas, sino que también incluye universidades latinoamericanas. Según el corpus, un estudiante chino elige el Máster Universitario Internacional en Estudios Contemporáneos de América Latina establecido por la Universidad Complutense de Madrid, la Universidad de Costa Rica y la Universidad de Uruguay.

Respecto a China, los títulos conjuntos se iniciaron en la década de 1980. En el siglo XXI, el ritmo de la implantación se ha acelerado. Entre ellos, la iniciativa de títulos conjuntos internacionales se desarrolla más rápido que los dos primeros y ha conseguido buenos logros en los últimos años. Por ejemplo: en octubre de 2007, 12 universidades ${ }^{12}$ de Beijing decidieron implantar títulos conjuntos internacionales para los estudiantes de postgrado en cooperación con universidades extranjeras. En junio de 2010, un total de 201 estudiantes de postgrado obtuvieron títulos conjuntos internacionales. (Xingwu \& Yong, 2011:4)

A medida que aumenta el alcance de este tipo de formación, aparecen cada día más modelos con innovación. Por ejemplo, la Universidad de Estudios Extranjeros de Guangdong (en adelante GDUFS) lleva muchos años explorando y mejorando el sistema internacional de educación de postgrado. En 2017, la Universidad aprovechó la gran variedad de especialidades relacionadas con lenguas extranjeras con las que cuenta y cofundó el título de postgrado de «Gestión e Innovación Internacional» con la Universidad de Maryland, EE. UU., recibiendo el apoyo total del Consejo Nacional de Becas de China. Este proyecto aplica el sistema académico «1+1+1». Es decir, los estudiantes estudian en GDUFS el primer año escolar. El segundo año, en la Universidad de Maryland. El tercer año, las dos universidades les ofrecerán oportunidades de hacer prácticas externas en las Naciones Unidas, el Banco Mundial, el Fondo Monetario Internacional, la Organización Mundial del Comercio y otras organizaciones internacionales. Los graduados recibirán sendos títulos de postgrado ${ }^{13}$.

Estas medidas pueden mejorar la cualidad integral de los posgraduados, promover el desarrollo de la educación de máster en las universidades, ampliar los intercambios internacionales y encontrar nuevas vías adecuadas para la formación de postgrado.

\section{Plan de estudios de máster universitario}

El plan de estudios incorpora plenamente los puntos clave del sistema de formación de la educación superior: asignaturas científicas, créditos flexibles, evaluaciones estrictas, etc. Conforme al Ministerio de Educación ${ }^{14}$, los estudios de máster comprenden entre 60 y 120 créditos europeos (entre 1 y 2 años). Si un alumno quiere obtener el título oficial, tiene que cumplir todos los requisitos establecidos por el plan de estudios y realizar los ejercicios correspondientes para la conclusión de la carrera. El corpus muestra que el $98 \%$ de los estudiantes chinos son capaces de completar todos los créditos en un año académico y recibir el título oficial. Justamente el corto plazo constituye un factor relevante para atraer a los chinos a estudiar en España, porque en China, generalmente

\footnotetext{
1212 universidades chinas son: la Universidad Médica de Beijing, la Academia de Cine de Beijing, la Universidad del Petróleo de Beijing, la Universidad Renmin de China, la Universidad de Tecnología Química de Beijing, la Universidad Central de Finanzas y Economía, la Universidad de Comercios Internacionales y Economía de la Capital, la Universidad de Comercios Internacionales y Economía, la Universidad de Estudios Extranjeros de Beijing, la Universidad de Lenguas y Culturas de Beijing, la Universidad Normal de Beijing y la Universidad Normal de la Capital.

13 http://yz.chsi.com.cn/kyzx/kyft/201709/20170913/1628092289.html

14 http://www.educacion.gob.es/boloniaensecundaria/o9-damos-respuesta1.htm\#pregunta2
} 
necesitan por lo menos 2 años para terminar el estudio de máster, según el Programa de Enseñanza de máster en China ${ }^{15}$.

En la actualidad, el plan de estudios varía según las universidades y carreras, y tiene muy buena acogida entre los estudiantes chinos. Según los certificados académicos personales, hay 4 modelos de diseño curricular:

Modelo 1: asignaturas obligatorias + optativas + trabajo fin de máster. Este es el modo más adoptado por las universidades, especialmente las carreras de ciencias, ciencias de la salud, ingenierías y arquitectura. Por ejemplo, máster universitario en Ingeniería Mecánica de la Universidad Politécnica de Madrid tiene 4 asignaturas obligatorias, 13 optativas y el trabajo fin de máster. Entonces, cabe preguntar: ¿una misma especialidad de distintas universidades tiene el mismo plan de estudio? La contestación es no. En el caso del máster en Pensamiento Español e Iberoamericano, la Universidad Complutense de Madrid ofrece 6 asignaturas obligatorias, 4 optativas y el trabajo fin de máster; la Universidad Autónoma de Madrid, 3 asignaturas obligatorias, 5 optativas, 1 práctica externa y el trabajo fin de máster. Véase la siguiente tabla:

\section{Tabla 4}

Asignaturas del máster en Pensamiento Español e Iberoamericano

\begin{tabular}{|c|c|c|c|c|c|}
\hline \multicolumn{3}{|c|}{ Universidad Complutense de Madrid } & \multicolumn{3}{|c|}{ Universidad Autónoma de Madrid } \\
\hline Tipo & Asignatura & Crédito & Tipo & Asignatura & Crédito \\
\hline \multirow{6}{*}{ Obligatoria } & $\begin{array}{l}\text { Genelogía y estructura } \\
\text { del espacio } \\
\text { iberoamericano }\end{array}$ & 4 & \multirow{6}{*}{ Obligatoria } & $\begin{array}{l}\text { De la monarquía hispana a } \\
\text { la comunidad de las } \\
\text { repúblicas (1500-2000) }\end{array}$ & 5 \\
\hline & $\begin{array}{l}\text { Filosofía, multicultu- } \\
\text { ralismo y postcolonia- } \\
\text { lidad iberoamericana }\end{array}$ & 4 & & $\begin{array}{l}\text { De la escuela de Salamanca } \\
\text { al modernismo (siglos XVI- } \\
\text { XIX) }\end{array}$ & 5 \\
\hline & $\begin{array}{l}\text { Humanismo, barroco e } \\
\text { ilustración en el } \\
\text { pensamiento hispano- } \\
\text { americano }\end{array}$ & 5 & & \multirow[t]{4}{*}{$\begin{array}{l}\text { Identidad y literatura en } \\
\text { Hispanoamérica }\end{array}$} & 5 \\
\hline & $\begin{array}{l}\text { El pensamiento ibero- } \\
\text { americano en el siglo } \\
\text { XIX }\end{array}$ & 5 & & & \\
\hline & $\begin{array}{l}\text { Ortega y su diálogo } \\
\text { con la filosofía de } \\
\text { Iberoamérica }\end{array}$ & 5 & & & \\
\hline & $\begin{array}{l}\text { Grandes pensadores } \\
\text { de América Latina }\end{array}$ & 5 & & & \\
\hline
\end{tabular}




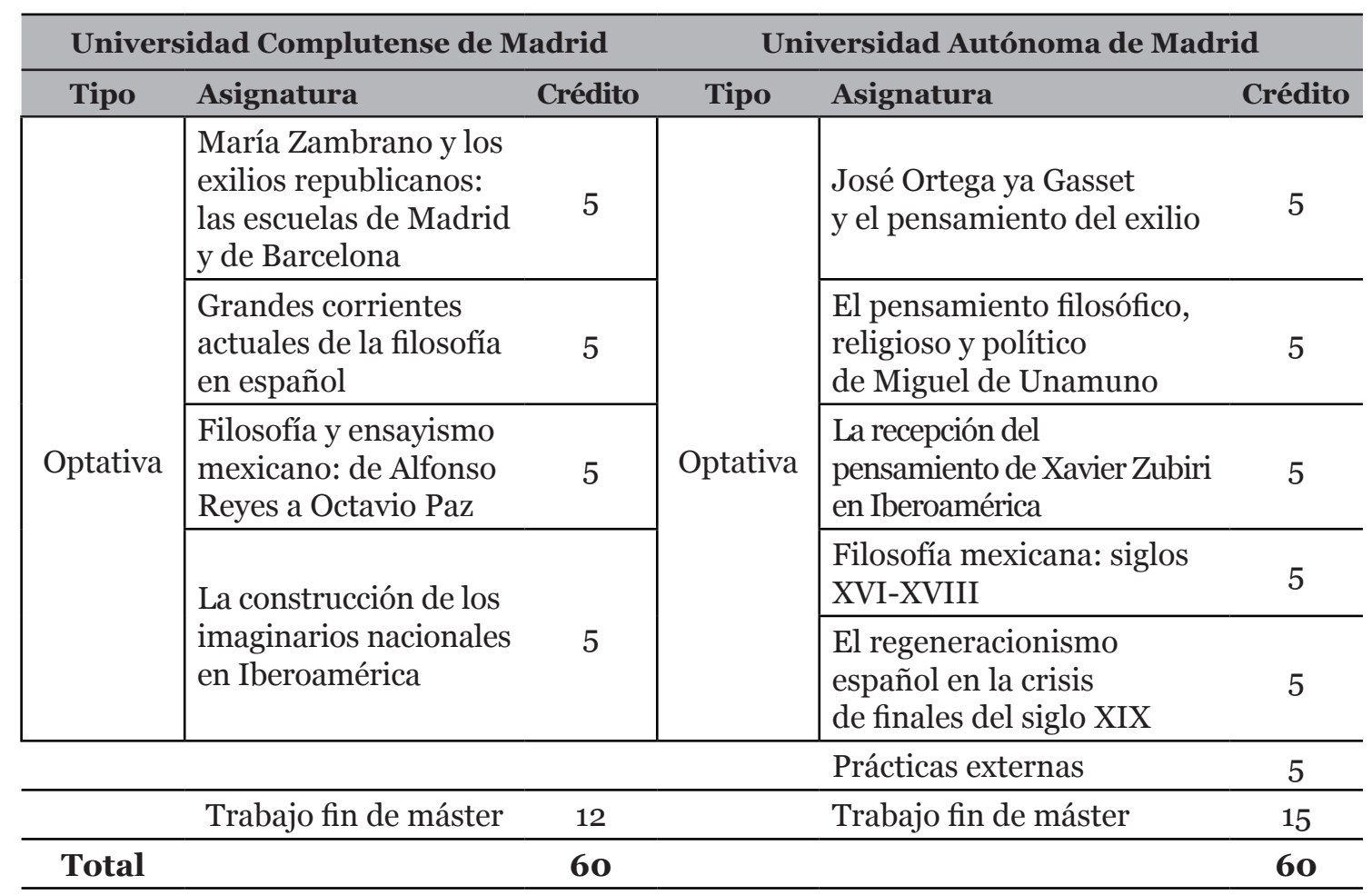

Además, existen sistemas curriculares que solo disponen de una asignatura obligatoria y el resto son optativas. Por ejemplo, el máster universitario en Cultura del Vino de la Universidad de Burgos. En el plan de estudios, el trabajo fin de máster y la asignatura «Vinos del mundo: tipos y propiedades» son obligatorios. Las otras 10 asignaturas son optativas: «Viticultura del siglo XXI», «Rutas del vino», «Entorno económico del sector vitivinícola», «Tradición e innovación enológica» etc.

Modelo 2: asignaturas obligatorias + práctica externa + trabajo fin de máster. Por ejemplo, máster universitario en Gestión e Investigación de la Comunicación Empresarial de la Universidad Rey Juan Carlos tiene un total de 11 asignaturas, de las cuales 7 asignaturas obligatorias deben ser completadas en un año académico. No hay exigencia para la fecha de terminación de las prácticas externas y el trabajo final. Ocurre lo mismo con el máster universitario en Comunicación Intercultural, Interpretación y Traducción en los Servicios Públicos de la Universidad de Alcalá. El alumnado tiene que terminar 8 asignaturas obligatorias en un año académico. Pero no hay demanda estricta de la fecha de finalización de la práctica externa y el trabajo final.

Modelo 3: asignaturas obligatorias + optativas + trabajo fin de máster + seminarios. Por ejemplo, el máster universitario en Teoría y Práctica del Proyecto de Arquitectura de la Universidad Politécnica de Cataluña tiene 5 materias obligatorias, 2 optativas y la tesina de fin de máster. Y además, el plan de estudios cuenta con el proyecto «Seminario de Crítica», que es obligatorio y tiene 10 créditos.

Modelo 4: asignaturas matriculadas + obligatorias + optativas + trabajo fin de máster. Por ejemplo, el máster universitario en Investigación en Periodismo: Discurso y Comunicación de la Universidad Complutense de Madrid aplica esta forma, cuyas materias y asignaturas están compuestas por 2 asignaturas matriculadas, 5 obligatorias, 6 optativas y tesina de fin de máster. 
En resumen, este diseño curricular facilita la opción de los estudiantes. Además, también facilita la evaluación final. Si un estudiante no presenta o tiene calificación de «suspenso» en una asignatura, tiene dos opciones. Por una parte, este puede presentarse en la siguiente convocatoria de la misma asignatura. Según el corpus, el $20 \%$ de ellos se han presentado en la segunda convocatoria y han logrado la calificación de «aprobado». Por otra parte, uno puede elegir otra asignatura y presentarse en la convocatoria. Esta forma mejora la eficiencia didáctica y la mayor parte de los estudiantes chinos puedan obtener el título dentro de un año.

\section{Conclusiones}

El análisis de los motivos por los cuales los estudiantes chinos eligen el máster en España y el descubrimiento de las características de la formación de máster en España y China son cuestiones muy significativas. Podemos ver que en el proceso de integración de la educación superior europea, la educación superior de España, con una larga historia, está desarrollándose con una gran vitalidad, especialmente los títulos de máster universitario que tienen acceso a conocimientos y habilidades estrechamente vinculados al mercado de trabajo, a corto plazo y con diseño curricular flexible, atraen cada vez más a estudiantes chinos para estudiar en España. Mientras tanto, durante los 40 años de la reforma y apertura en China, ha habido un gran desarrollo en la educación de máster. En particular, se ha registrado un progreso considerable en la cooperación internacional en los últimos años. En la actualidad, nos enfrentamos a un reto: cómo hacer que la educación de máster sea más adecuada para el mercado de trabajo. Por eso, la educación de máster actual de España puede ser una referencia valiosa.

\section{Referencias bibliográficas}

Claudio Quiroga, G. (2017). Intercambios educativos entre China y España en la Universidad plataforma de acercamiento entre los dos países. Anuario jurídico y económico escurialense, 475-498.

Coello de Portugal, J.M.. (2015). Acerca de la memoria anual de seguimiento de las titulaciones de grado y máster: aprendiendo a mejorar la calidad académica y la gestión de la enseñanza. Revista general de derecho constitucional, 21.

Crespo Navarro, E. (2010). máster universitario en integración regional: un título conjunto fruto de la cooperación interuniversitaria España-Centroamérica, Transatlántica de educación, 8, 93-98

Jianlin, Y. (2009). The Trend of Structural Adjustment of Postgraduate Education in China from the Perspective of International Comparison. Academic Degrees \& Graduate Education, 8, 66-71.

Ministerio de Educación Cultura y Deporte (ed.). (2016). Las cifras de la educación en España. Estadísticas e indicadores. Estadística, 237-252.

Ministry of Education, National Development and Reform Commission, Ministry of Finance (2013). Opinions on Deepening the Reform of Postgraduate Education. 
Mingyi, S. (2010). Education Reform of Finnish Doctor in Bologna and Its Enlightenment. Academic Degrees \& Graduate Education, 1, 73-77.

Xingwu, G. \& Yong, H. (2011). The current situation, problems and measures of joint training of graduate students in China and abroad in Beijing. Academic Degrees \& Graduate Education, 12, 4-10.

Xiuyan, W. (2013). Measures and Enlightenment of Spanish Graduate Education Reform in Bologna Process. Education and Examinations, 4, 78-81. 